TITLE:

\title{
Effect of tensile force on the mechanical behavior of actin filaments.
}

\section{$\operatorname{AUTHOR}(S)$ :}

Matsushita, Shinji; Inoue, Yasuhiro; Hojo, Masaki; Sokabe, Masahiro; Adachi, Taiji

\section{CITATION:}

Matsushita, Shinji ... [et al]. Effect of tensile force on the mechanical behavior of actin filaments.. Journal of biomechanics 2011, 44(9): 17761781

ISSUE DATE:

2011-06-03

URL:

http://hdl.handle.net/2433/152437

\section{RIGHT:}

@ 2011 Elsevier Ltd.; この論文は出版社版でありません。引用の際には 出版社版をご確認ご利用ください。; This is not the published version. Please cite only the published version. 


\section{Effect of tensile force on the mechanical behavior of actin filaments}

2

3

Shinji Matsushita ${ }^{1,2,3}$, Yasuhiro Inoue ${ }^{1,2,3}$, Masaki Hojo ${ }^{4}$, Masahiro Sokabe ${ }^{5,6}$ and Taiji Adachi ${ }^{1,2,3}$

${ }^{1}$ Department of Biomechanics, Research Center for Nano Medical Engineering, Institute for Frontier Medical Sciences, Kyoto University, Sakyo, Kyoto 606-8507, Japan

${ }^{2}$ Department of Micro Engineering, Graduate School of Engineering, Kyoto University Sakyo, Kyoto 606-8501, Japan

${ }^{3}$ Computational Cell Biomechanics Team, VCAD System Research Program, RIKEN Hirosawa, Wako 351-0198, Japan

${ }^{4}$ Department of Mechanical Engineering and Science, Graduate School of Engineering, Kyoto University, Sakyo, Kyoto 606-8501, Japan

${ }^{5}$ Department of Physiology, Graduate School of Medicine, Nagoya University, 65 Tsurumai, Nagoya 466-8550, Japan

${ }^{6}$ FIRST Research Center for Innovative Nanobiodevice, Nagoya University, Furo-cho, Nagoya 464-8603, Japan

\section{Corresponding Author:}

Taiji Adachi, Ph.D.

\section{Mailing Address:}

Department of Biomechanics

Research Center for Nano Medical Engineering

Institute for Frontier Medical Sciences

Kyoto University

53 Kawahara-cho, Shogoin, Sakyo, Kyoto 606-8507, Japan

Tel \& Fax: +81 (75)751-4853

E-mail: adachi@frontier.kyoto-u.ac.jp

Submitted to the Journal of Biomechanics 


\section{Abstract}

Actin filaments are the most abundant components of the cellular cytoskeleton, and play critical roles in various cellular functions such as migration, division and shape control. In these activities, mechanical tension causes structural changes in the double-helical structure of the actin filament, which is a key modulator of cytoskeletal reorganization. This study performed large-scale molecular dynamics (MD) and steered MD simulations to quantitatively analyze the effects of tensile force on the mechanical behavior of actin filaments. The results revealed that when a tensile force of $200 \mathrm{pN}$ was applied to a filament consisting of 14 actin subunits, the twist angle of the filament decreased by approximately 20 degrees, corresponding to a rotation of approximately -2 degrees per subunit, representing a critical structural change in actin filaments. Based on these structural changes, the variance in filament length and twist angle was found to decrease, leading to increases in extensional and torsional stiffness. Torsional stiffness increased significantly under the tensile condition, and the ratio of filament stiffness under tensile force to that under no external force increased significantly on longer temporal scales. The results obtained from this study contribute to the understanding of mechanochemical interactions concerning actin dynamics, showing that increased tensile force in the filament prevents actin regulatory proteins from binding to the filament.

Keywords: Actin filament, Tensile force, Mechanical properties, Mechano-chemical interactions, Steered molecular dynamics simulation, Computational biomechanics, Cell mechanics 


\section{Introduction}

(1)

The major components of the actin cytoskeleton, actin filaments, play critical roles in

various cellular functions, such as migration, division and shape control (Svitkina et al., 1997;

Watanabe and Mitchison, 2002; Pollard and Berro, 2009; Pollard and Borisy, 2003; Adachi et al., 2009). In these activities, the actin cytoskeleton undergoes dynamic rearrangements governed by mechanical and biochemical factors (Arber et al., 1998; Isenberg et al., 1980;

Pollard and Cooper, 1986; Theriot and Mitchison, 1991). In particular, changes in mechanical conditions within the cells and in their surrounding environment are key regulatory factors affecting the global reorganization of the actin cytoskeleton (Naruse and Sokabe, 1993;

Neidlinger-Wilke et al., 2001; Sato et al., 2005; Sato et al., 2000; Yamamoto et al., 2006). In this reorganization process, microscopic mechanical stretching, twisting and bending cause structural changes at the molecular level in single actin filaments having a double-helical structure (Holmes et al., 1990; Oda et al., 2009). This structural modulation is critical for inducing local cytoskeletal reorganization by interacting with a variety of biochemical factors and triggering the binding of actin regulatory proteins to the filaments (Hayakawa et al., 2008; McGough et al., 1997; Prochniewicz et al., 2005). Investigation of the molecular mechanisms underlying how mechanical forces such as tension (Ishijima et al., 1991; Shimozawa and Ishiwata, 2009) and torsional moment modulate the mechanical behaviors of a single actin filament is thus important (Tsuda et al., 1996). Analysis of the mechanical behaviors of actin filaments at the molecular structural level is performed using numerical simulations based on the molecular dynamics (MD) method 
1 (Chu and Voth, 2005, 2006; Pfaendtner et al., 2010). The steered MD (SMD) method

2 (Isralewitz et al., 2001) enables control of the positions and/or velocities of some specific

3 atoms by applying external steering forces in the appropriate direction. The SMD method is

4 widely used to investigate the mechanical behaviors of proteins, such as stretching of the

5 extracellular matrix (Krammer et al., 1999) and muscle proteins (Craig et al., 2002),

6 binding/unbinding of protein-substrate complexes (Isralewitz et al., 1997; Lu et al., 1998) and

7 adhesion proteins (Bayas et al., 2003; Vogel and Sheetz, 2006) and dissociation of phosphate

8 from G-actin (Wriggers and Schulten, 1997, 1999). Thus, using the SMD method, we can

9 quantitatively analyze the structural dynamics of actin molecules under external forces

10 relevant to biological problems at the molecular level.

Our previous study (Matsushita et al., 2010) quantitatively evaluated the extensional and

torsional stiffness of a single actin filament under no external forces based on an analysis of

thermal fluctuations in the molecular structure using the MD method. The present study used the SMD method to investigate the effects of tensile force on mechanical behaviors of the filament. We first applied a tensile force to the molecular structure of the actin filament that was pre-equilibrated under a no-force condition. We then compared the mechanical behavior of the filament under tensile force to that under no external force.

\section{Methods}

\section{Simulation of the filament under no external force}


1 ionic solvate was obtained using the same procedure applied in our previous studies

2 (Matsushita et al., 2010). The original actin filament structure is available from the Protein

3 Data Bank (PDB code: 1MVW) (Chen et al., 2002; Holmes et al., 1990). As the actin subunits

4 in filaments under tensile force are mainly bound to ADP, ADP was placed in the individual

5 actin subunits in the filament, in which the initial coordinates of ADP were determined from

6 the ADP-bound monomer structure (PDB code: 1J6Z) (Otterbein et al., 2001). The entire

7 structure was solvated in a water box of dimensions $117 \times 118 \times 473 \AA$, to which $\mathrm{Na}^{+}$and $\mathrm{Cl}^{-}$

8 counter ions were added at a concentration of $30 \mathrm{mM}$. This system was equilibrated in an

9 NPT ensemble (pressure $=1 \mathrm{~atm}$, temperature $=310 \mathrm{~K}$ ) by performing MD simulation for 20

10 ns using NAMD 2.6 (Kale et al., 1999) with the CHARMM27 force field for proteins

11 (MacKerell et al., 1998) and the TIP3P model for water (Jorgensen et al., 1983). We applied

periodic boundary conditions for simulations where van der Waals interactions were

calculated with a cut-off distance of $13 \AA$ and electrostatic interactions were calculated using

the particle mesh Ewald method (Darden et al., 1993). Free dynamics simulation under no

external force was then performed for $12 \mathrm{~ns}$ to analyze the mechanical behavior of the

filament.

Simulation of the filament under tensile force

SMD simulation under constant tensile conditions was performed by applying the minus-end (Fig. 1B). Total constant tensile force to the filament was set as $F=200 \mathrm{pN}$, a 
1 value smaller than the breaking force of the actin-actin bonds, which ranges from 320 to 600

2 pN (Tsuda et al., 1996).

SMD simulation under the tensile condition was performed in the same environmental

4

5

\section{Results}

\section{Changes in molecular structure of actin filament}

Quantitative analysis of structural changes in the actin filament under different mechanical conditions was performed considering the rotational and longitudinal motions,

which are critical structural motions of the filament. Rotational motion is particularly important for interaction of the actin filament with various biochemical factors (McCullough

et al., 2008). In this study, filament length $L(t)$ and twist angle of the filament $\Theta(t)$ (Fig. 1C) were defined as follows (Matsushita et al., 2010):

$L(t)=z_{\text {plus }}(t)-z_{\text {minus }}(t)$

where $z_{\text {plus }}(t)$ is the position on the $z$-axis of the center of mass of the $\mathrm{G}$-actins $\mathrm{G}_{13}$ and $\mathrm{G}_{14}$ at the plus-end, and $z_{\text {minus }}(t)$ is that of the G-actins $\mathrm{G}_{1}$ and $\mathrm{G}_{2}$ at the minus-end. 
$1 \quad \Theta(t)=\cos ^{-1}\left(\frac{\boldsymbol{n}_{\text {plus }}(t)}{\left|\boldsymbol{n}_{\text {plus }}(t)\right|} \cdot \frac{\boldsymbol{n}_{\text {minus }}(t)}{\left|\boldsymbol{n}_{\text {minus }}(t)\right|}\right)$,

2 where

$3 \quad \boldsymbol{n}_{\text {minus }}(t)=\boldsymbol{P}_{\mathrm{G} 2}(t)-\boldsymbol{P}_{\mathrm{G} 1}(t)$,

$4 \quad \boldsymbol{n}_{\mathrm{plus}}(t)=\boldsymbol{P}_{\mathrm{G} 14}(t)-\boldsymbol{P}_{\mathrm{G} 13}(t)$,

5 and $\boldsymbol{P}_{\mathrm{Gi}}(t)$ is the position vector of the center of mass of the $\mathrm{G}$-actin $\mathrm{G}_{\mathrm{i}}$ projected onto the $x-y$

6 plane.

We monitored longitudinal thermal fluctuations in the actin filament under no external or tensile force, as shown in Figure 2A, in which changes in filament length $L(t)$ are plotted at

9 intervals of 1 ps. For quantitative measurement of the elongation by external tensile force, the probability distribution of $L(t)$ and the approximated normal distribution curve were plotted as

11 solid lines at intervals of $0.01 \AA$ and broken lines, respectively (Fig. 2B). Figure 2B shows that the average $\left(\langle L(t)\rangle_{12 \mathrm{~ns}}\right)$ and standard deviation $\left(\sqrt{\left\langle\Delta L^{2}(t)\right\rangle_{12 \mathrm{~ns}}}\right.$

$\left.13=\sqrt{\left.\left\langle(L(t)-<L(t)\rangle_{12 \mathrm{~ns}}\right)^{2}\right\rangle_{12 \mathrm{~ns}}}\right)$ of length over the 12-ns period were $326.7 \pm 0.6 \AA$ and 327.3 $14 \pm 0.5 \AA$ under no-force and tensile conditions, respectively, indicating that the elongation of approximately $0.6 \AA$ corresponds to an extensional strain of approximately $0.2 \%$. This was in agreement with the calculated strain of $0.2 \%$ based on extensional stiffness of $3.1 \mathrm{~N} / \mathrm{m}$ (Kojima et al., 1994) and an applied external tensile force $F=200 \mathrm{pN}$. 
1 angle $\Theta(t)$. As shown in these figures, the average $\left(\langle\Theta(t)\rangle_{12 \mathrm{~ns}}\right)$ and standard deviation

$2\left(\sqrt{\left\langle\Delta \Theta^{2}(t)\right\rangle_{12 \mathrm{~ns}}}=\sqrt{\left\langle\left(\Theta(t)-\langle\Theta(t)\rangle_{12 \mathrm{~ns}}\right)^{2}\right\rangle_{12 \mathrm{~ns}}}\right)$ of the twist angle over the 12-ns period

3 under no-force and tensile conditions were 179.2 \pm 6.9 degrees and $159.0 \pm 3.3$ degrees,

4 respectively. By applying a tensile force of $200 \mathrm{pN}$ to the filament, the twist angle decreased

5 by an average of 20.2 degrees, probably due to the structural feature of the right-handed

6 double helix, where extensional motions of the tensile force induced coupled torsional

7 motions. On simplifying the filament to a homogeneous rod model of a circular cross-section

8 with a diameter equal to the magnitude of vector $\boldsymbol{n}_{\text {minus }}(t)$ defined in Eq. (3), the 20.2 degrees

9 change in twist angle corresponds to a shear strain of $1.6 \%$ on the outer surface of the rod.

10 The magnitude of the $1.6 \%$ shear strain may be significant enough to induce changes in the

11 mechanical behavior of the filaments.

\section{Changes in extensional and torsional stiffness}

We estimated the apparent extensional stiffness $\left(K_{\text {ext }}^{\Delta t}(t)\right)$ and torsional stiffness $\left(K_{\text {tor }}^{\Delta t}(t)\right)$

of the actin filament from the variances of the filament length $L(t)$ and twist angle $\Theta(t)$ during is expressed as follows:

$$
\frac{1}{2} k_{\mathrm{ext}}^{\Delta t}(t)<\left(L(t)-<L(t)>_{\Delta t}\right)^{2}>_{\Delta t}=\frac{1}{2} k_{\mathrm{B}} T
$$

$$
\frac{1}{2} k_{\mathrm{tor}}^{\Delta t}(t)<\left(\Theta(t)-<\Theta(t)>_{\Delta t}\right)^{2}>_{\Delta t}=\frac{1}{2} k_{\mathrm{B}} T,
$$

where $k_{\mathrm{ext}}^{\Delta t}(t)$ and $k_{\mathrm{tor}}^{\Delta t}(t)$ are the extensional and torsional spring constants, respectively, $k_{\mathrm{B}}$ 
1 a time period $\left(t-\frac{\Delta t}{2} \leq t<t+\frac{\Delta t}{2}\right)$. If the potential energy that determines mechanical

2 behavior of the filament can be approximated as a harmonic potential in the vicinity of a

3 certain equilibrium point at a given temperature, the law of equipartition of energy is satisfied irrespective of whether tensile force is applied to the actin filaments. From the spring

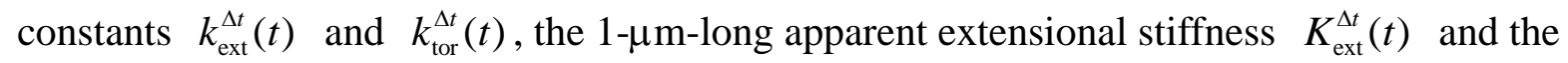
apparent torsional stiffness per unit length of filament $K_{\text {tor }}^{\Delta t}(t)$ are given by

$$
\begin{aligned}
& K_{\mathrm{ext}}^{\Delta t}(t)=\frac{\langle L(t)\rangle_{\Delta t}}{1 \mu \mathrm{m}} k_{\mathrm{ext}}^{\Delta t}(t), \\
& K_{\mathrm{tor}}^{\Delta t}(t)=\langle L(t)\rangle_{\Delta t} k_{\mathrm{tor}}^{\Delta \mathrm{t}}(t) .
\end{aligned}
$$

Figure 3A shows the change over time in the extensional stiffness $K_{\text {ext }}^{\Delta t}(t)$ plotted at intervals of $1 \mathrm{ps}$, determined for each sampling-window duration $(\Delta t=0.5,1.0,2.0,4.0$ and $8.0 \mathrm{~ns})$. To show the dependence of apparent stiffness on the sampling-window duration, the average and standard deviation of $K_{\mathrm{ext}}^{\Delta t}(t)$ for each sampling-window duration are plotted against the sampling-window duration $\Delta t$ (Fig. 3B). According to previous reports (Matsushita et al., 2010), $K_{\mathrm{ext}}^{\Delta t}(t)$ tended to decrease with increasing $\Delta t$ and to converge to a value. Similarly, as shown in Figure 3C and Figure 3D, torsional stiffness $K_{\text {tor }}^{\Delta t}(t)$ decreased with an increase in $\Delta t$ and converged to a value.

As shown in Figure 3B and Figure 3D, both extensional and torsional stiffness increased with the application of tensile force to the actin filaments indicated by blue and red lines. To quantitatively analyze an increase in stiffness due to tensile force, the ratios of filament stiffness under tensile force to that under no external force, $K_{\alpha}^{\text {tensile force }} / K_{\alpha}^{\text {no external force }}(\alpha=$ ext, 
1 tor), are plotted in Figure 4. The ratio of torsional stiffness exhibited a large increase with

2 increasing sampling-window duration and is expected to converge on a certain value, given a

3 sampling-window duration long enough for the filament stiffness to converge (Fig. 3D).

4 When compared to the longest sampling-window duration $\Delta t=8.0$ [ns], torsional stiffness of

5 the filament under tensile force was 3.5-fold larger than that under no external force. In

6 contrast, the ratio of extensional stiffness exhibited no significant change. The increase in

$7 \quad$ stiffness due to tensile force was thus found to differ between extensional and torsional

8 stiffness.

Increases in torsional stiffness may be attributable to changes in the twist structure of the

filament. The applied tensile force decreased the twist angle of the filament by approximately $20^{\circ}$, indicating that tensile force tightened the double-helical structure laterally. This constrains the rotational motions of the filament, resulting in increased torsional stiffness. In contrast, extensional stiffness showed no significant change, as filament length and longitudinal motions of the filament were constant (Fig. 2B).

\section{Discussion}

This study quantitatively analyzed the effects of tensile force on the extensional and torsional stiffness of actin filaments by performing MD and SMD simulations. When a tensile force $F$ of $200 \mathrm{pN}$ was applied to a filament consisting of 14 actin subunits, the twist angle decreased by approximately 20 degrees (Fig. 2D), representing a significant structural change

21 for the actin filament. Given the structural changes, variances in filament length and twist 
1 angle due to thermal fluctuations were found to decrease, leading to increases in extensional

2 and torsional stiffness (Fig. 3B, 3D). Torsional stiffness increased significantly under the

3 tensile force, and the ratio of filament stiffness under tensile force to that under no external

4 force $\left(K_{\text {tor }}^{\text {tenile force }} / K_{\text {tor }}^{\text {no external force }}\right)$ increased significantly with longer sampling-window duration 5 (Fig. 4).

In order to focus on the fundamental characteristic of the dependence of the tensile force on extensional and torsional displacement, we chose the tensile condition of $F=200 \mathrm{pN}$ as a typical example, as well as the no external force condition $F=0 \mathrm{pN}$. Using stiffness under the two tensile conditions of $F=0 \mathrm{pN}$ and $200 \mathrm{pN}$, we can approximate the mechanical behavior of the filament under tensile force less than $\sim 320 \mathrm{pN}$ before breaking. Under the tensile force $F=200 \mathrm{pN}$, the extensional stiffness $K_{\mathrm{ext}}^{200 \mathrm{pN}}$ increased 1.2-fold compared to that under tensile force $F=0 \mathrm{pN}, K_{\mathrm{ext}}^{0 \mathrm{pN}} \quad$ (Fig. 4). This indicates that the tensile force-extensional displacement relation exhibits nonlinear behavior. Using stiffness and displacement under the two tensile conditions, we can fit the force-displacement function as a 3rd-order polynomial. An understanding of the tensile force-torsional displacement relationship requires quantitative evaluation of the tensile force-torsional displacement coupling stiffness in the vicinity of equilibrium point $K_{\text {couple }}=F /\langle\Delta \Theta\rangle$, where $F$ is tensile force and $\langle\Delta \Theta\rangle$ is average torsional displacement angle. This will be our next challenge, as further discussion is necessary regarding the law of equipartition of energy in consideration of the extension and torsion coupling stiffness $K_{\text {couple }}$. We investigated the molecular behavior of actin filaments from mechanical and 
1 structural perspectives and studied the mechanical properties involved. A number of reports

2 applying the SMD method have successfully analyzed the mechanical behaviors of various

3 biomolecules under external forces (Craig et al., 2002; Lu et al., 1998; Vogel and Sheetz,

4 2006), where changes in molecular structure are studied during transition processes

$5 \quad$ characterized by a thermal non-equilibrium state. In contrast, this study performed SMD

6 simulation to observe thermally equilibrated molecular behaviors under constant tensile

7 conditions, enabling quantitative analysis of thermal fluctuations of the molecules as well as

8 global structural changes, such as changes in filament length and twist angle. As these thermal

9 fluctuations in actin filaments are among the factors determining macroscopic mechanical

10 properties, analysis of thermally equilibrated molecular behaviors is necessary to achieve a

11 fundamental understanding of the mechanical properties of actin filaments.

Actin subunits are generally bound to ADP, ADP/Pi and ATP molecules and have three

nucleotide states. The dependence of these three nucleotide states on the stiffness of filaments our purpose of investigating the effect of tensile force on the mechanical behavior of tensile force are mainly bound to ADP during dynamic cellular activities. For example, in the process of cell migration, ATP- and ADP/Pi-actin filaments exist in the vicinity of the leading 
1 actin subunits bound to ADP is thus essential to achieving an understanding of the mechanical

2 behavior of filaments under tensile force.

Changes in mechanical behavior induced by external tensile force will significantly

4 affect interactions between the actin filament and various biochemical factors. Microscopic

5 tensile force along the single filament, generated by macroscopic intracellular contractile

6 forces in the actin stress fibers (Neidlinger-Wilke et al., 2001), plays an essential role in

7 interactions with a variety of actin-binding proteins, such as cofilin (Hayakawa et al., 2008).

8 In contrast, actin-binding proteins such as myosin II play a role in generating microscopic

9 mechanical tensile force, which in turn induces macroscopic contractile forces in dynamic

10 cellular processes such as cell migration (Adachi et al., 2009). The interaction between

11 mechanical and biochemical factors is thus critical for various cellular activities. However,

little is known about the molecular mechanisms underlying interactions between tension and actin-binding proteins. Further insights into the physical mechanisms of mechanochemical and chemomechanical coupling as they concern actin dynamics would thus be helpful. shown in this study suggests a strong relationship to the affinity for actin regulatory proteins such as cofilin. Cofilin is an actin depolymerizing and severing factor that binds along the length of the filament and increases torsional (Prochniewicz et al., 2005) and bending 
1 actin subunits per crossover (Meberg et al., 1998). When cofilin binds to the actin filament,

2 the filament is locally twisted by approximately 5 degrees per subunit, from -167 degrees to

$3-162$ degrees, and the number of subunits per crossover decreases to approximately 10

$4 \quad$ (Bamburg et al., 1999).

In this study, by applying a tensile force of $200 \mathrm{pN}$ to filaments with a half-turn structure consisting of 14 subunits, the twist angle was decreased by approximately 20 degrees, corresponding to a rotation of approximately -2 degrees, i.e., from -165 degrees to -167 degrees per subunit, in a direction opposite to that of the structural change when cofilin binds to the filament (Fig. 5). If the affinity of cofilin depends on the twist angle between subunits, the results obtained in this study suggest that tensile force applied to the filament prevents cofilin from binding to the filament. In addition, application of tensile force increases the torsional stiffness; that is, the variance of the twist angle decreases. The structure of proteins is not stable in one static structure, but dynamically transitions through various local metastable structures because of thermal fluctuations. Furthermore, various biochemical interactions such as protein binding occur stochastically in the thermal fluctuations. The decrease in twisting fluctuations under tensile conditions thus suggests that the binding probability of cofilin also decreases because of the decrease in twist angle. Quantitative investigation of the binding affinities of actin regulatory proteins, including cofilin, will necessitate analysis of the interactions between actin filaments and actin regulatory proteins based on free energy estimation, which will in turn enable quantification of the effects of tensile force on mechanical behaviors of the filament. 
2 Conflict of Interest

$3 \quad$ None.

4

\section{Acknowledgements}

6

This research was partly supported by a Grant-in-aid for Specially Promoted Research

7 (20001007) from the Ministry of Education, Culture, Sports, Science and Technology

8 (MEXT). The computing system was provided by Research and Development of the

9 Next-Generation Integrated Simulation of Living Matter, a part of the Development and Use

of the Next-Generation Supercomputer Project. Shinji Matsushita was supported by the Japan

11 Society for the Promotion of Science (JSPS) as a JSPS Fellow.

12

13

14 


\section{References}

Adachi, T., Okeyo, K.O., Shitagawa, Y., Hojo, M., 2009, Strain field in actin filament network in lamellipodia of migrating cells: Implication for network reorganization. Journal of Biomechanics 42, 297-302.

Arber, S., Barbayannis, F.A., Hanser, H., Schneider, C., Stanyon, C.A., Bernard, O., Caroni, P., 1998, Regulation of actin dynamics through phosphorylation of cofilin by LIM-kinase. Nature 393, 805-809.

Bamburg, J.R., McGough, A., Ono, S., 1999, Putting a new twist on actin: ADF/cofilins modulate actin dynamics. Trends in Cell Biology 9, 364-370.

Bayas, M.V., Schulten, K., Leckband, D., 2003, Forced detachment of the CD2-CD58 complex. Biophysical Journal 84, 2223-2233.

Chen, L.F., Winkler, H., Reedy, M.K., Reedy, M.C., Taylor, K.A., 2002, Molecular modeling of averaged rigor crossbridges from tomograms of insect flight muscle. Journal of Structural Biology 138, 92-104.

Chu, J.W., Voth, G.A., 2005, Allostery of actin filaments: Molecular dynamics simulations and coarse-grained analysis. Proceedings of the National Academy of Sciences of the United States of America 102, 13111-13116.

Chu, J.W., Voth, G.A., 2006, Coarse-grained modeling of the actin filament derived from atomistic-scale simulations. Biophysical Journal 90, 1572-1582.

Craig, D., Gao, M., Schulten, K., Vogel, V., 2002, Steered molecular dynamics simulations of the extracellular matrix protein fibronectin. Molecular Biology of the Cell 13, 1137.

Darden, T., York, D., Pedersen, L., 1993, Paticle mesh ewald - an N.log(N) method for ewald sums in large systems. Journal of Chemical Physics 98, 10089-10092.

Hayakawa, K., Tatsumi, H., Sokabe, M., 2008, Actin stress fibers transmit and focus force to activate mechanosensitive channels. Journal of Cell Science 121, 496-503.

Holmes, K.C., Popp, D., Gebhard, W., Kabsch, W., 1990, Atomic model of the actin filament. Nature 347, 44-49.

Isenberg, G., Aebi, U., Pollard, T.D., 1980, An actin-binding protein from acanthamoeba regulates actin filament polymerization and interactions. Nature 288, 455-459.

Ishijima, A., Doi, T., Sakurada, K., Yanagida, T., 1991, Sub-piconewton force fluctuations of actomyosin invitro. Nature 352, 301-306.

Isralewitz, B., Gao, M., Schulten, K., 2001, Steered molecular dynamics and mechanical functions of proteins. Current Opinion in Structural Biology 11, 224-230.

Isralewitz, B., Izrailev, S., Schulten, K., 1997, Binding pathway of retinal to bacterio-opsin: A prediction by molecular dynamics simulations. Biophysical Journal 73, 2972-2979.

Jorgensen, W.L., Chandrasekhar, J., Madura, J.D., Impey, R.W., Klein, M.L., 1983, Comparison of simple potemtial functions for simulating liquid water. Journal of Chemical Physics 79, 926-935.

Kale, L., Skeel, R., Bhandarkar, M., Brunner, R., Gursoy, A., Krawetz, N., Phillips, J., Shinozaki, A., Varadarajan, K., Schulten, K., 1999, NAMD2: Greater scalability for parallel molecular dynamics. Journal of Computational Physics 151, 283-312. 
Kojima, H., Ishijima, A., Yanagida, T., 1994, Direct measurement of stiffness of single actin-filaments with and without tropomyosin by in-vitro nanomanipulation. Proceedings of the National Academy of Sciences of the United States of America 91, 12962-12966.

Krammer, A., Lu, H., Isralewitz, B., Schulten, K., Vogel, V., 1999, Forced unfolding of the fibronectin type III module reveals a tensile molecular recognition switch. Proceedings of the National Academy of Sciences of the United States of America 96, 1351-1356.

Lu, H., Isralewitz, B., Krammer, A., Vogel, V., Schulten, K., 1998, Unfolding of titin immunoglobulin domains by steered molecular dynamics simulation. Biophysical Journal 75, 662-671.

MacKerell, A.D., Bashford, D., Bellott, M., Dunbrack, R.L., Evanseck, J.D., Field, M.J., Fischer, S., Gao, J., Guo, H., Ha, S., Joseph-McCarthy, D., Kuchnir, L., Kuczera, K., Lau, F.T.K., Mattos, C., Michnick, S., Ngo, T., Nguyen, D.T., Prodhom, B., Reiher, W.E., Roux, B., Schlenkrich, M., Smith, J.C., Stote, R., Straub, J., Watanabe, M., Wiorkiewicz-Kuczera, J., Yin, D., Karplus, M., 1998, All-atom empirical potential for molecular modeling and dynamics studies of proteins. Journal of Physical Chemistry B 102, 3586-3616.

Matsushita, S., Adachi, T., Inoue, Y., Hojo, M., Sokabe, M., 2010, Evaluation of extensional and torsional stiffness of single actin filaments by molecular dynamics analysis. Journal of Biomechanics 43, 3162-3167.

McCullough, B.R., Blanchoin, L., Martiel, J.L., De La Cruz, E.M., 2008, Cofilin increases the bending flexibility of actin filaments: Implications for severing and cell mechanics. Journal of Molecular Biology 381, 550-558.

McGough, A., Pope, B., Chiu, W., Weeds, A., 1997, Cofilin changes the twist of F-actin: Implications for actin filament dynamics and cellular function. Journal of Cell Biology 138, 771-781.

Meberg, P.J., Ono, S., Minamide, L.S., Takahashi, M., Bamburg, J.R., 1998, Actin depolymerizing factor and cofilin phosphorylation dynamics: Response to signals that regulate neurite extension. Cell Motility and the Cytoskeleton 39, 172-190.

Naruse, K., Sokabe, M., 1993, Involvement of strech-activated ion channels in Ca2+ mobilization to mechanical strech in endothelial-cells. American Journal of Physiology 264, C1037-C1044.

Neidlinger-Wilke, C., Grood, E.S., Wang, J.H.C., Brand, R.A., Claes, L., 2001, Cell alignment is induced by cyclic changes in cell length: studies of cells grown in cyclically stretched substrates. Journal of Orthopaedic Research 19, 286-293.

Oda, T., Iwasa, M., Aihara, T., Maeda, Y., Narita, A., 2009, The nature of the globular-to fibrous-actin transition. Nature 457, 441-445.

Otterbein, L.R., Graceffa, P., Dominguez, R., 2001, The crystal structure of uncomplexed actin in the ADP state. Science 293, 708-711.

Pfaendtner, J., Lyman, E., Pollard, T.D., Voth, G.A., 2010, Structure and dynamics of the actin filament. Journal of Molecular Biology 396, 252-263. 
Pollard, T.D., Berro, J., 2009, Mathematical models and simulations of cellular processes based on actin filaments. Journal of Biological Chemistry 284, 5433-5437.

Pollard, T.D., Borisy, G.G., 2003, Cellular motility driven by assembly and disassembly of actin filaments. Cell 112, 453-465.

Pollard, T.D., Cooper, J.A., 1986, Actin and actin-binding proteins - a critical-evaluation of mechanism and functions. Annual Review of Biochemistry 55, 987-1035.

Prochniewicz, E., Janson, N., Thomas, D.D., De La Cruz, E.M., 2005, Cofilin increases the torsional flexibility and dynamics of actin filaments. Journal of Molecular Biology 353, 990-1000.

Sato, K., Adachi, T., Matsuo, M., Tomita, Y., 2005, Quantitative evaluation of threshold fiber strain that induces reorganization of cytoskeletal actin fiber structure in osteoblastic cells. Journal of Biomechanics 38, 1895-1901.

Sato, M., Nagayama, K., Kataoka, N., Sasaki, M., Hane, K., 2000, Local mechanical properties measured by atomic force microscopy for cultured bovine endothelial cells exposed to shear stress. Journal of Biomechanics 33, 127-135.

Shimozawa, T., Ishiwata, S., 2009, Mechanical distortion of single actin filaments induced by external force: detection by fluorescence imaging. Biophysical Journal 96, 1036-1044.

Svitkina, T.M., Verkhovsky, A.B., McQuade, K.M., Borisy, G.G., 1997, Analysis of the actin-myosin II system in fish epidermal keratocytes: Mechanism of cell body translocation. Journal of Cell Biology 139, 397-415.

Theriot, J.A., Mitchison, T.J., 1991, Actin microfilament dynamics in locomoting cells. Nature 352, 126-131.

Tsuda, Y., Yasutake, H., Ishijima, A., Yanagida, T., 1996, Torsional rigidity of single actin filaments and actin-actin bond breaking force under torsion measured directly by in vitro micromanipulation. Proceedings of the National Academy of Sciences of the United States of America 93, 12937-12942.

Vogel, V., Sheetz, M., 2006, Local force and geometry sensing regulate cell functions. Nature Reviews Molecular Cell Biology 7, 265-275.

Watanabe, N., Mitchison, T.J., 2002, Single-molecule speckle analysis of actin filament turnover in lamellipodia. Science 295, 1083-1086.

Wriggers, W., Schulten, K., 1997, Stability and dynamics of G-actin: Back-door water diffusion and behavior of a subdomain 3/4 loop. Biophysical Journal 73, 624-639.

Wriggers, W., Schulten, K., 1999, Investigating a back door mechanism of actin phosphate release by steered molecular dynamics. Proteins-Structure Function and Genetics 35, 262-273.

Yamamoto, K., Sokabe, T., Matsumoto, T., Yoshimura, K., Shibata, M., Ohura, N., Fukuda, T., Sato, T., Sekine, K., Kato, S., Isshiki, M., Fujita, T., Kobayashi, M., Kawamura, K., Masuda, H., Kamiya, A., Ando, J., 2006, Impaired flow-dependent control of vascular tone and remodeling in P2X4-deficient mice. Nature Medicine 12, 133-137. 
A

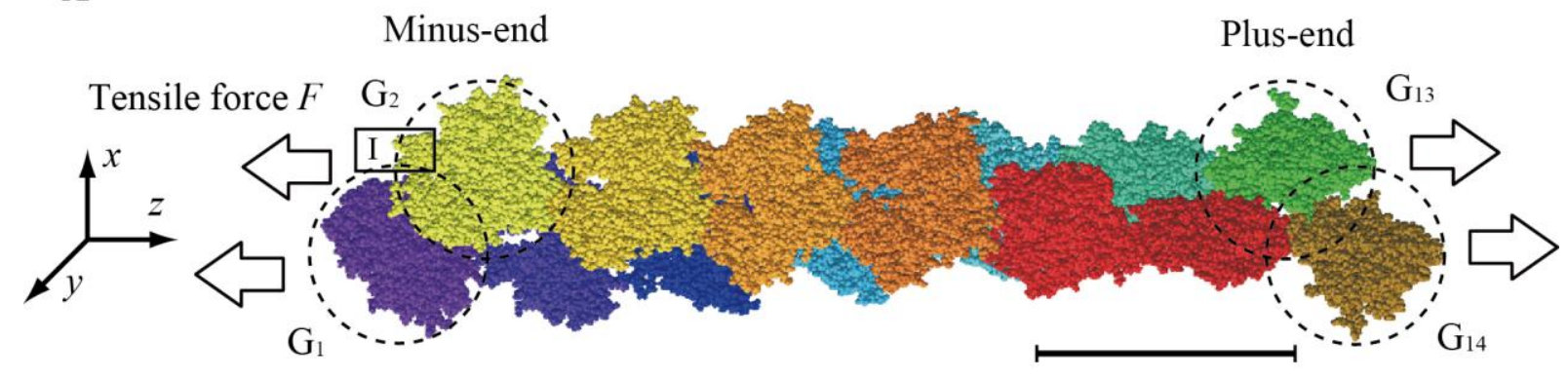

B

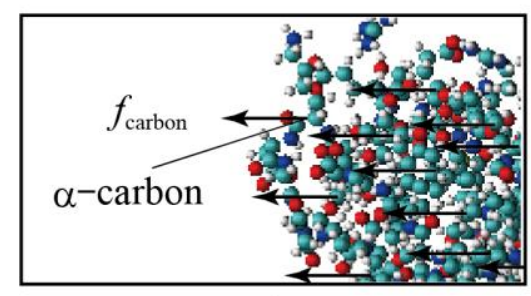

Region I
C

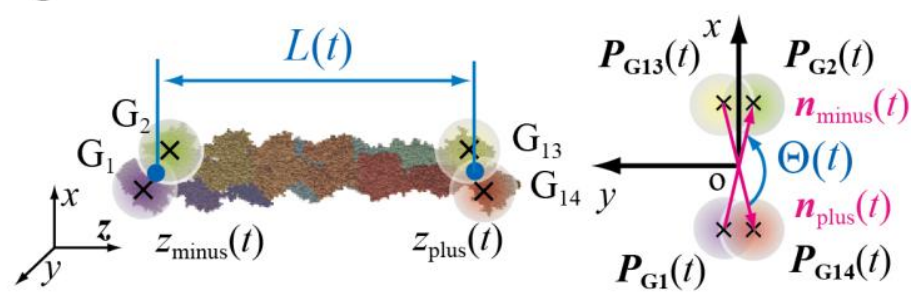

1

Figure 1: Actin filament structure model analyzed in the SMD simulation. A) Double-helical structure of an actin filament consisting of 14 actin subunits. Each actin subunit is numbered from the minus-end as $G_{1}, G_{2}, \ldots, G_{14}$. Scale bar represents $100 \AA$. B) An image of Region I in (A) magnified 6-fold. In SMD simulations, constant tensile forces were applied to the

filament at the $\alpha$-carbons in the actin subunits $G_{1}, G_{2}, G_{13}$ and $G_{14}$ at both ends. C) Filament length $L(t)$ was defined by Eq. (1) as the distance between the plus and minus ends of the

9 filament. Twist angle $\Theta(t)$ was defined by Eq. (2) as the angle between the vectors $\boldsymbol{n}_{\text {plus }}$ and $\boldsymbol{n}_{\text {minus }}$ of the plus- and minus-ends, projected onto the $x-y$ plane. 
A

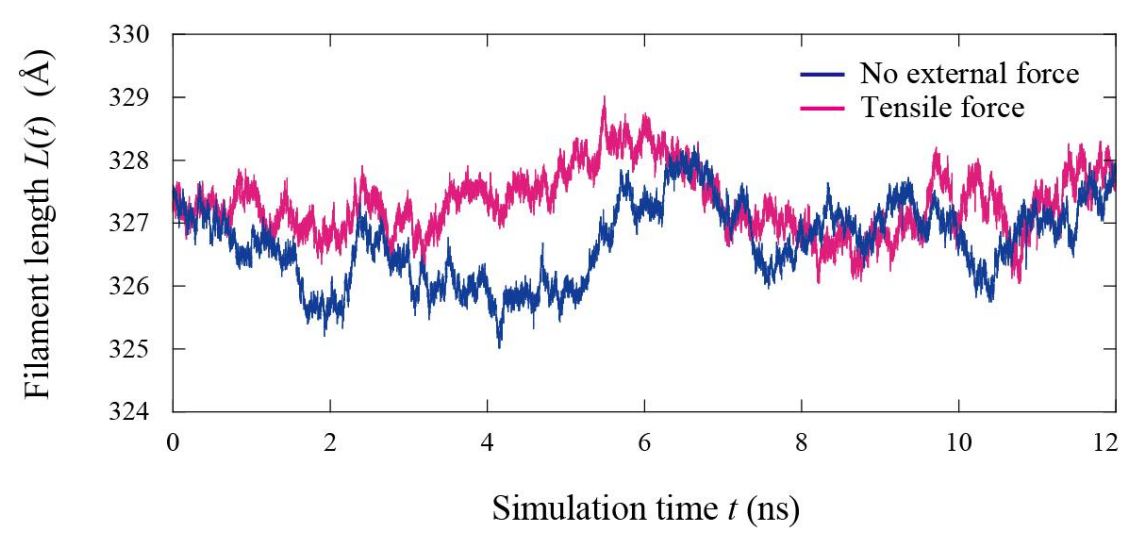

C

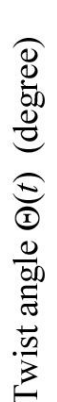

1

2

3

4

5

6

7

Simulation time $t(\mathrm{~ns})$ condition.

\section{B}

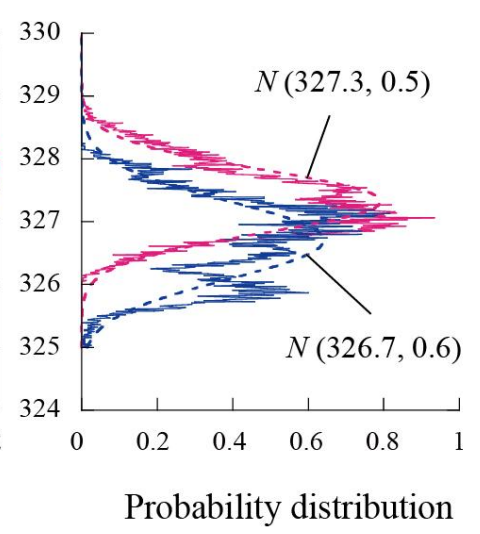

D

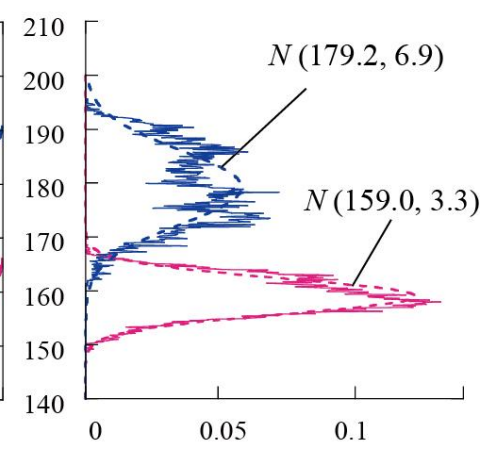

Probability distribution

Figure 2: Structural changes during 12-ns simulations under no-force and tensile conditions.

A) Change in filament length $L(t)$. B) Average filament length is $326.7 \AA$ under the no-force condition and 327.3 $\AA$ under the tensile condition. C) Change in twist angle $\Theta(t)$. D) Average twist angle is 179.2 degrees under the no-force condition and 159.0 degrees under the tensile 
A

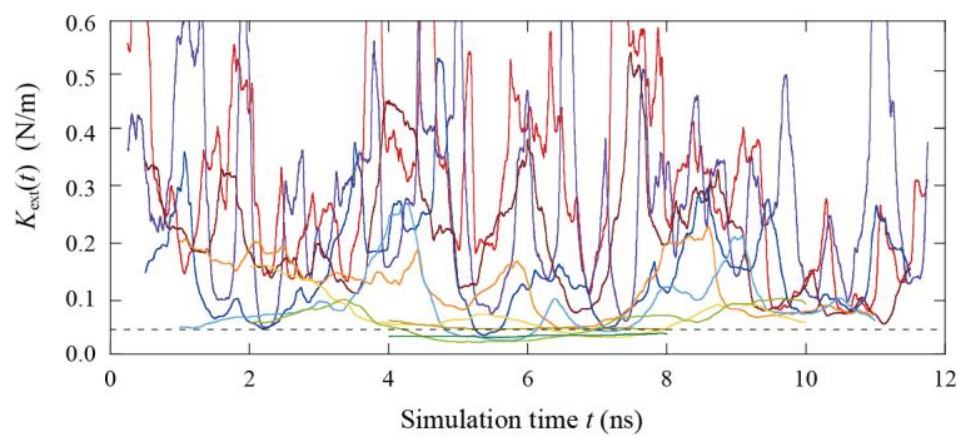

C

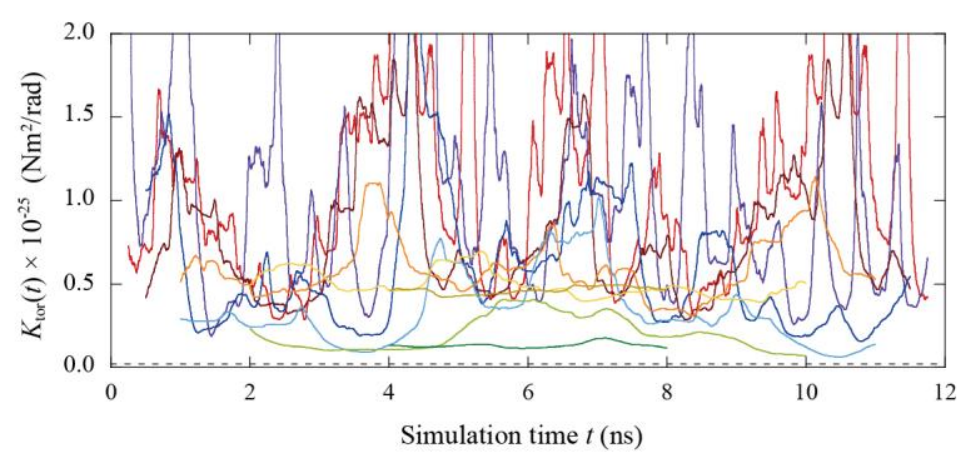

\section{B}

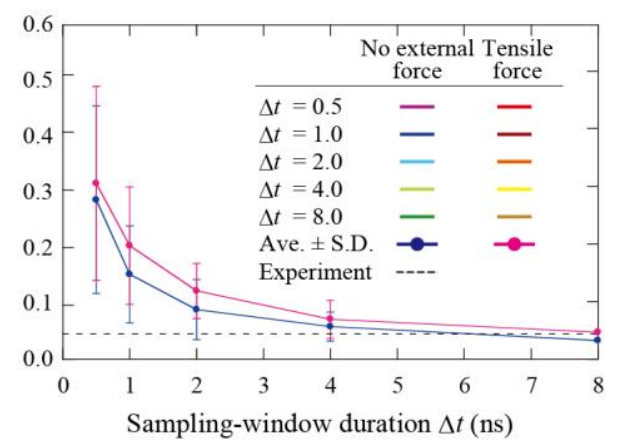

D

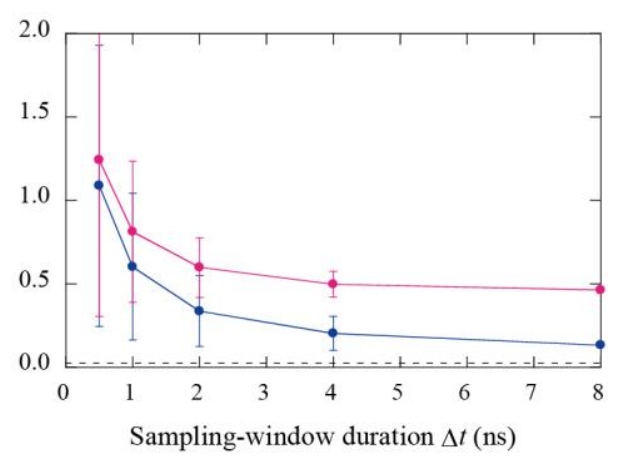

$3 \quad$ Figure 3: Extensional and torsional stiffness under the no-force and tensile conditions determined for each sampling-window duration $\Delta t$. Experimentally determined extensional

$5 \quad$ stiffness (Kojima et al., 1994) and torsional stiffness (Prochniewicz et al., 2005) are

6 represented by dashed lines. A) Change in extensional stiffness $K_{\text {ext }}^{\Delta t}(t)$. B) Average

7 extensional stiffness $K_{\mathrm{ext}}^{\Delta t}(t)$ for each sampling-window duration $\Delta t$. C) Change in torsional

8 stiffness $K_{\text {tor }}^{\Delta t}(t)$. D) Average torsional stiffness $K_{\text {tor }}^{\Delta t}(t)$ for each sampling-window duration

$9 \Delta t$ 


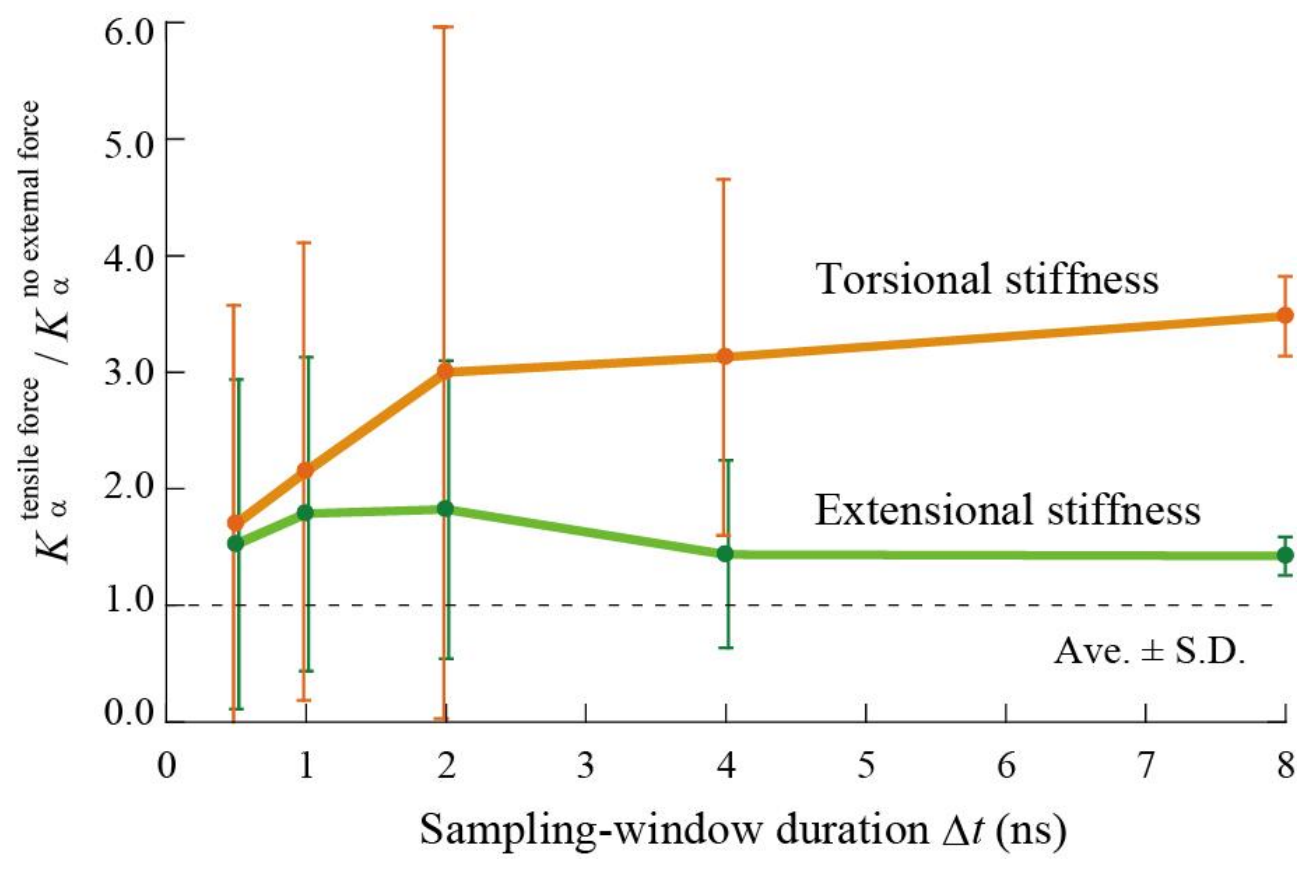

2

$3 \quad$ Figure 4: Ratios of extensional and torsional stiffness under tensile force to that under no

4 external force [ $K_{\alpha}^{\text {tensile force }} / K_{\alpha}^{\text {no external force }}(\alpha=$ ext, tor $)$ ], and their dependence on

5 sampling-window duration $\Delta t$.

6

7 


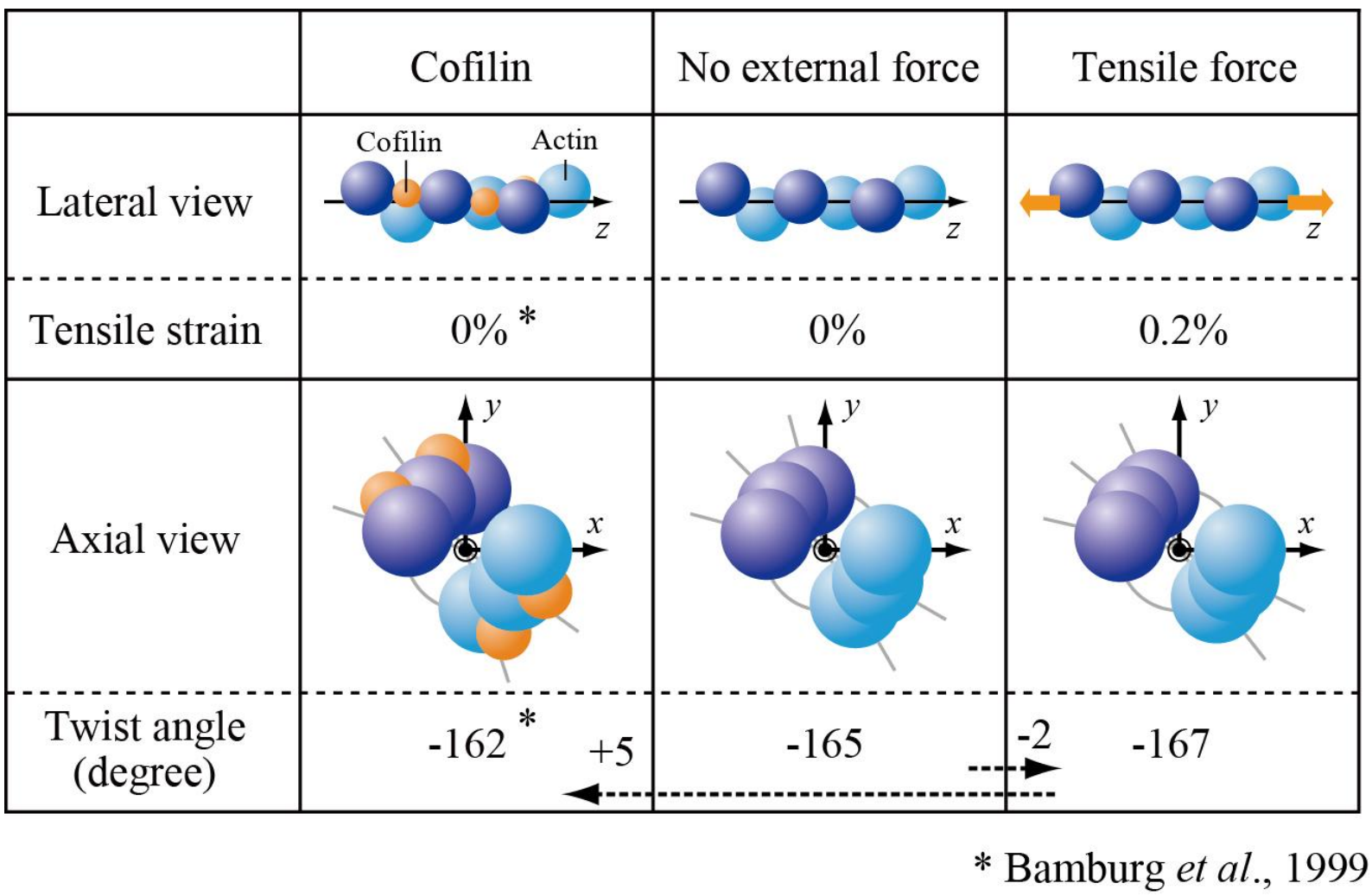

3 Figure 5: Longitudinal and rotational motions of an actin filament induced by tensile force

4 and binding of cofilins. When tensile force is applied to the actin filament, the filament is

5 twisted by approximately -2 degrees per subunit from its structure under no external force.

6 When cofilins bind to the actin filament, the filament is twisted by approximately +5 degrees

7 per subunit from its structure under tensile force. 